\section{Prevalence of Clonal Mast Cell Disorders in Patients Presenting With Hymenoptera Venom Anaphylaxis Might Be Higher Than Expected}

\author{
Vázquez-Revuelta $\mathrm{P}^{1, *}$, González-de-Olano $\mathrm{D}^{1, *}$ \\ ${ }^{1}$ Allergy Department, Hospital Universitario Ramón y Cajal, \\ Madrid, Spain \\ *Both authors contributed equally to this manuscript and should \\ be considered first authors
}

J Investig Allergol Clin Immunol 2018; Vol. 28(3): 193-194 doi: 10.18176/jiaci.0233

Key words: Clonal mast cell activation syndromes. Hymenoptera venom anaphylaxis. Mastocytosis. Prevalence.

Palabras clave: Síndromes de activación mastocitaria. Anafilaxia veneno himenópteros. Mastocitosis. Prevalencia.

Anaphylactic reactions after hymenoptera stings are probably the leading cause of anaphylaxis in patients with mast cell disorders, especially indolent systemic mastocytosis without skin involvement (ISMs-) or with clonal mast cell activation syndrome (c-MCAS) [1,2]. According to published data, mastocytosis accounts for $1 \%-7 \%$ of all cases of hymenoptera venom anaphylaxis (HVA) [3]. In most patients, the anaphylactic reaction after exposure to hymenoptera venom might be the sole manifestation of their disease $[2,4]$. Although the confirmed diagnosis of mastocytosis requires a complete bone marrow study, the European Competence Network on Mastocytosis recommends using the so-called REMA score (Red Española de Mastocitosis [Spanish Mastocytosis Network]) as a useful screening tool for predicting the presence of clonal mast cells before the bone marrow examination is performed [5]. The REMA score analyzes 4 independent factors, namely, gender, cardiovascular symptoms, absence of pruritus/urticaria/angioedema, and baseline serum tryptase level. A score $\geq 2$ predicts clonality with high values of specificity in patients in the absence of skin lesions of mastocytosis. This score is even more sensitive for patients with HVA [6]. Therefore, the REMA score can facilitate the diagnosis of clonal mast cell disorders $[7,8]$.

In an effort to discover the prevalence of possible clonal mast cell disorders among patients presenting with HVA according to the REMA score, we retrospectively analyzed data from all patients who had experienced anaphylactic reactions after hymenoptera stings and were referred to the allergy department of our hospital, which is located in an urban area in the center of Spain, where the frequency of hymenoptera stings is low. During April 2017, we queried all electronic records of patients who had been evaluated at our department since electronic medical records were available (June 1999) until the date of the search. We used the current Boolean search software (Excalibur, Retrieval Ware/Convera, Inc.) and introduced the terms honeybee, wasp, and hymenoptera. We obtained a total of 1924 results that were carefully analyzed. Symptoms following the hymenoptera sting were recorded, and only 174 patients with hymenoptera venom anaphylaxis according to accepted criteria [9] were selected. At the time the search was made, 9 patients $(6.4 \%)$ had already been diagnosed with mastocytosis after a bone marrow study. In 32 patients, clinical and analytical variables were not registered and, thus, the REMA score could not be calculated. The score was applied to the remaining patients with HVA, who were divided into 2 groups according to the result. Both groups were subsequently analyzed (Table). Our study is limited by its retrospective design.

Confirmation of the diagnosis of mast cell disorder requires a complete bone marrow study and fulfilment of diagnostic criteria for cytology, histology, and flow cytometry immunophenotyping using specific gating strategies for the detection of bone marrow mast cells that are present at low frequencies, as well as the study of the KIT mutation in purified mast cells [10]. Mast cells are infrequent in patients with ISMs- or c-MCAS, in whom HVA is, by far, the most frequent trigger of anaphylaxis $[2,11]$. Therefore, specialized centers with more sensitive techniques are needed to reach the final diagnosis in most cases. On this basis, the REMA score has become an extremely helpful tool that easily predicts clonality in a subset of patients who will require further specific management. In the present study, $23.2 \%$ of patients with HVA had a positive REMA score. Clinical features and gender distribution were similar to those of the mastocytosis group,

Table. Baseline Characteristics of the Population ${ }^{a}$

\begin{tabular}{lccccc}
\hline & Mastocytosis $\left(\mathrm{n}=9^{\mathrm{b}}\right)$ & $P$ value & Score $\geq 2(\mathrm{n}=33)$ & $P$ Value & Score $<2(\mathrm{n}=100)$ \\
\hline Male & $7 / 9(78)$ & NS & $23 / 33(70)$ & .03 & $49 / 100(49)$ \\
Age & $55(30-71)$ & NS & $47(13-87)$ & NS & $49(10-78)$ \\
Total IgE, kU/L & $73.7(16-202)$ & NS & $109^{\mathrm{c}}(0-330)$ & NS & $191(2-2001)$ \\
Baseline tryptase,$\mu \mathrm{g} / \mathrm{L}$ & $12.1(1-17.9)$ & 0.01 & $5.7(1-10)$ & NS & $5.2(1-21)$
\end{tabular}

Abbreviation: REMA, Red Española de Mastocitosis (Spanish Mastocytosis Network).

${ }^{a}$ Results expressed as number of patients/total patients studied (percentage) for categorical variables and median (range) for continuous variables.

${ }^{\mathrm{b}} 7 / 8$ had a REMA score $\geq 2$. The remaining patient had cutaneous mastocytosis.

'Only 28/33 patients had total IgE determination available. 
with the exception of baseline serum tryptase level, which has already been reported to be frequently low in patients with ISMs-, and c-MCAS [7]. The utility of the REMA score has been demonstrated [7] and validated [8]. When the score is applied considering only patients with HVA, sensitivity reaches $92 \%$ [6], with a positive predictive value of $89 \%$ [7]. If we apply this percentage to the patients in our series with a REMA score $\geq 2$, assuming that our prevalence of cases would be the same as that of the study in which the REMA score was performed, 29/33 would probably be real mastocytosis (ie, $20.4 \%$ ). This figure, along with the $6.4 \%$ previously diagnosed at our center after the bone marrow mast cell biopsy, would increase the final rate of clonal mast cell disorder to $26.8 \%$, which is higher than the $1 \%-7 \%$ reported elsewhere [4].

A clear association between HVA and clonal mast cells has been reported [12]. This association is even stronger in ISMsand c-MCAS patients, and the frequency of hymenoptera as a trigger of anaphylaxis may reach up to $50 \%$ [2]. In fact, since these patients usually present a low mast cell burden, with fewer symptoms associated with release of mast cell mediators between the acute episodes and normal baseline serum tryptase values [7], they may often be underdiagnosed. Identification of this subset of at-risk patients is necessary, since they present more adverse effects during venom immunotherapy and may need to extend venom immunotherapy beyond the standard duration [13]. Although a bone marrow mast cell biopsy is mandatory to confirm the diagnosis of mast cell disorder, application of the REMA score to all patients presenting with anaphylaxis after a hymenoptera sting in the absence of skin lesions might be helpful — and economical - for identification of clonal mast cell disorder, which might be more frequent than expected among patients with HVA.

\section{Acknowledgments}

We thank Sarah Barnes for her technical assistance with the revision of the English version of the manuscript.

\section{Funding}

This study was supported by a grant from the Sociedad Española Alergia e Inmunología Clínica 2014.

\section{Conflicts of Interest}

The authors declare that they have no conflicts of interest.

\section{References}

1. Gonzalez-de-Olano D, Alvarez-Twose I. Insights in Anaphylaxis and Clonal Mast Cell Disorders. Front Immunol. 2017:8:792.

2. Matito A, Alvarez-Twose I, Morgado JM, Sanchez-Munoz L, Orfao A, Escribano L. Anaphylaxis as a clinical manifestation of clonal mast cell disorders. Curr Allergy Asthma Rep. 2014; 14:450.

3. Bonadonna P, Lombardo C, Zanotti R. Mastocytosis and allergic diseases. J Investig Allergol Clin Immunol. 2014;24(5):288-97.

4. Bonadonna P, Bonifacio M, Lombardo C, Zanotti R. Hymenoptera Allergy and Mast Cell Activation Syndromes. Curr Allergy Asthma Rep. 2016;16:5.
5. Valent P, Escribano L, Broesby-Olsen S, Hartmann K, Grattan C, Brockow K, et al. Proposed diagnostic algorithm for patients with suspected mastocytosis: a proposal of the European Competence Network on Mastocytosis. Allergy. 2014;69:1267-74.

6. Alvarez-Twose I, Zanotti R, Gonzalez-de-Olano D, Bonadonna $P$, Vega A, Matito A, et al. Nonaggressive systemic mastocytosis (SM) without skin lesions associated with insect-induced anaphylaxis shows unique features versus other indolent SM. J Allergy Clin Immunol. 2014;133:520-8.

7. Alvarez-Twose I, Gonzalez de Olano D, Sanchez-Munoz L, Matito A, Esteban-Lopez MI, Vega A, et al. Clinical, biological, and molecular characteristics of clonal mast cell disorders presenting with systemic mast cell activation symptoms. J Allergy Clin Immunol. 2010;125:1269-78 e2.

8. Alvarez-Twose I, Gonzalez-de-Olano D, Sanchez-Munoz L, Matito A, Jara-Acevedo M, Teodosio C, et al. Validation of the REMA Score for Predicting Mast Cell Clonality and Systemic Mastocytosis in Patients with Systemic Mast Cell Activation Symptoms. Int Arch Allergy Immunol. 2012;157:275-80.

9. Sampson HA, Munoz-Furlong $A$, Campbell RL, Adkinson NF, Jr., Bock SA, Branum A, et al. Second symposium on the definition and management of anaphylaxis: summary report-Second National Institute of Allergy and Infectious Disease/ Food Allergy and Anaphylaxis Network symposium. J Allergy Clin Immunol. 2006;117:391.

10. Gonzalez-de-Olano D, Matito A, Orfao A, Escribano L. Advances in the understanding and clinical management of mastocytosis and clonal mast cell activation syndromes. F1000Res. 2016;5:2666.

11. Alfaya Arias T, Soriano Gómis V, Soto Mera T, Vega Castro A, Vega Gutiérrez JM, Alonso Llamazares A, et al. Key Issues in Hymenoptera Venom Allergy: An Update. J Investig Allergol Clin Immunol. 2017;27(1):19-31.

12. Gonzalez de Olano D, de la Hoz Caballer B, Nuñez Lopez R, Sanchez Munoz L, Cuevas Agustin M, Dieguez MC, et al. Prevalence of allergy and anaphylactic symptoms in 210 adult and pediatric patients with mastocytosis in Spain: a study of the Spanish network on mastocytosis (REMA). Clin Exp Allergy. 2007;37:1547-55.

13. Gonzalez-de-Olano D, Alvarez-Twose I, Vega A, Orfao A, Escribano L. Venom immunotherapy in patients with mastocytosis and hymenoptera venom anaphylaxis. Immunotherapy. 2011;3:637-51.

1 Manuscript received December 23, 2017; accepted for publication January 24, 2018.

David González-de-Olano Hospital Universitario Ramón y Cajal Servicio de Alergia Cta. Colmenar Viejo km 9,1 28034, Madrid (Spain) E-mail: dgolano@yahoo.es 